PUBLICATIONS DE L'INSTITUT MATHÉMATIQUE

Nouvelle série, tome 76(90) (2004), 41-55

\title{
THE SPECTRAL MEAN SQUARE OF HECKE $L$-FUNCTIONS ON THE CRITICAL LINE
}

\author{
M. Jutila
}

Communicated by Aleksandar Ivić

\begin{abstract}
The Hecke $L$-function $H_{j}(s)$ attached to the $j$ th Maass form for the full modular group is estimated in the mean square over a spectral interval for $s=\frac{1}{2}+i t$. As a corollary, we obtain the estimate $H_{j}\left(\frac{1}{2}+i t\right) \ll$ $t^{1 / 3+\varepsilon}$ for $t \gg \kappa_{j}^{3 / 2}$, where $1 / 4+\kappa_{j}^{2}$ is the respective $j$ th eigenvalue of the hyperbolic Laplacian. This extends a result due to T. Meurman.
\end{abstract}

\section{Introduction}

We adopt the notation of the monograph [Mo] of Y. Motohashi; thus the Hecke $L$-function $H_{j}(s)$, defined for $\operatorname{Re} s>1$ by the Dirichlet series

$$
H_{j}(s)=\sum_{n=1}^{\infty} t_{j}(n) n^{-s}
$$

is related to the $j$ th Maass form $\psi_{j}$ for the full modular group, and the coefficients $t_{j}(n)$ are the corresponding Hecke eigenvalues for $\psi_{j}$. The form $\psi_{j}$ is an eigenfunction of the hyperbolic Laplacian, and the respective eigenvalue is written as $1 / 4+\kappa_{j}^{2}$ with $\kappa_{j}>0$. In [JM1] it was proved - as a generalization of the main result in [J4] concerning the case $t=0$ - that

$$
\sum_{\left|\kappa_{j}-K\right| \leqslant K^{1 / 3}} \alpha_{j}\left|H_{j}\left(\frac{1}{2}+i t\right)\right|^{4} \ll K^{4 / 3+\varepsilon} \text { for } 0 \leqslant t \leqslant K^{2 / 3},
$$

2000 Mathematics Subject Classification. Primary 11F66; Secondary 11M41.

Key words and phrases. automorphic $L$-functions, spectral theory. 
where $\alpha_{j}=\left|\rho_{j}\right|^{2} / \cosh \left(\pi \kappa_{j}\right)$ with $\rho_{j}$ the first Fourier coefficient of $\psi_{j}$. Since it is known that $\alpha_{j} \gg \kappa_{j}^{-\varepsilon}$ (see [Iw1]), this implies that

$$
H_{j}\left(\frac{1}{2}+i t\right) \ll \kappa_{j}^{1 / 3+\varepsilon} \text { for } 0 \leqslant t \leqslant \kappa_{j}^{2 / 3}
$$

in the case $t=0$, this was first shown by A. Ivic [Iv2]. The "trivial" bound is $H_{j}\left(\frac{1}{2}+i t\right) \ll\left(t+\kappa_{j}\right)^{1 / 2+\varepsilon}$ which follows from the functional equation of $H_{j}(s)$ by the convexity principle, so that the estimate (1.1) may be viewed as a subconvexity bound. More generally, one may conjecture the validity of the hybrid subconvexity estimate

$$
H_{j}\left(\frac{1}{2}+i t\right) \ll\left(\kappa_{j}+t\right)^{1 / 3+\varepsilon} \text { for } t \geqslant 0
$$

This has been proved by T. Meurman [Me2] for $t \gg \kappa_{j}^{3}$, but in view (1.1) the case $\kappa_{j}^{2 / 3} \ll t \ll \kappa_{j}^{3}$ still remains open. It is our purpose to shrink this problematic range to $\kappa_{j}^{2 / 3} \ll t \ll \kappa_{j}^{3 / 2}$ as a corollary of our main theorem. Finally, in [JM2], even this gap is filled, and the conjecture (1.2) is thus verified. It should be noted that the same estimate without $1 / 3$ in the exponent would be an analogue of Lindelöf's hypothesis, and this is of course the ultimate goal in this context.

As a notational convention, we let $\varepsilon$ denote generally a small positive constant, not necessarily the same at each occurrence, which may depend on other $\varepsilon$-numbers, whereas $\delta$ will stand for a small positive constant which will be fixed throughout that context where it is introduced.

ThEOREM. For $t \geqslant 0$ and $1 \ll G \ll K$, we have

$$
\sum_{K \leqslant \kappa_{j} \leqslant K+G} \alpha_{j}\left|H_{j}\left(\frac{1}{2}+i t\right)\right|^{2} \ll\left(G K+t^{2 / 3}\right)^{1+\varepsilon}
$$

Choosing $G=1$, we obtain immediately the following corollary.

Corollary. For $t \gg \kappa_{j}^{3 / 2}$, we have $H_{j}\left(\frac{1}{2}+i t\right) \ll t^{1 / 3+\varepsilon}$.

For orientation, we note that a standard argument based on the approximate functional equation for $H_{j}(s)$ and the spectral large sieve (Lemma 4 below) gives the bound $\ll(G K+t)^{1+\varepsilon}$ for the sum in (1.3), so that the case $t \ll K^{1+\varepsilon}$ is anyway clear. The remaining case $t \gg K^{1+\varepsilon}$ will be divided into two subcases,

$$
\begin{gathered}
K^{1+\varepsilon} \ll t \ll K^{3+\varepsilon}, \\
t \gg K^{3+\varepsilon},
\end{gathered}
$$

which will be treated by different arguments. Namely, in the case (1.4), we are going to apply the Bruggeman-Kuznetsov Spectral-Kloosterman sum formula and van 
der Corput's method, while the main tools in the case (1.5) will be certain transformation formulae for Dirichlet polynomials (analogous to those in [J1]), the method of "overlapping intervals", the spectral large sieve, and an arithmetic lemma from the Bombieri-Iwaniec method. The transformation device and the last mentioned lemma are common features of the present paper with [J2], where an alternative proof of Iwaniec's well-known estimate for the fourth moment of the zeta-function over a short interval on the critical line was given.

Though the arguments in the cases (1.4) and (1.5) will be different, the starting point is nevertheless the same: by the approximate functional equation for $H_{j}(s)$, say in the form of the Reflection Principle due to K. Ramachandra (see [Iv1, Sec. 4.4]), we may reduce the proof of (1.3) to showing that

$$
\sum_{K \leqslant \kappa_{j} \leqslant K+G} \alpha_{j}\left|S_{j}\right|^{2} \ll\left(G K+t^{2 / 3}\right)^{1+\varepsilon},
$$

where

$$
S_{j}=\sum_{M \leqslant m \leqslant M^{\prime}} t_{j}(m) m^{-1 / 2-i t}
$$

with $M<M^{\prime} \leqslant 2 M$ and $M \ll t$. Since (1.6) follows immediately from the spectral large sieve inequality if $M \ll\left(G K+t^{2 / 3}\right)^{1+\varepsilon}$, we may suppose henceforth that

$$
\left(G K+t^{2 / 3}\right)^{1+\varepsilon} \ll M \ll t .
$$

\section{The case (1.4)}

It suffices to prove (1.6) in the special case

$$
G=t^{2 / 3} K^{-1-\delta}+K^{\delta},
$$

where $\delta>0$ is a small positive constant, for the general assertion then follows on subdividing the range for $\kappa_{j}$ into subranges of length about (2.1) if the original range is longer, applying then (1.6) to each of these, and adding finally the estimates. Also, an estimate for the weighted sum

$$
S=\sum_{j=1}^{\infty} \alpha_{j} h\left(\kappa_{j}\right)\left|S_{j}\right|^{2}
$$

where

$$
h(r)=K^{-2}\left(r^{2}+\frac{1}{4}\right)\left\{\exp \left(-\left(\frac{r-K}{G}\right)^{2}\right)+\exp \left(-\left(\frac{r+K}{G}\right)^{2}\right)\right\},
$$

entails the same estimate for the sum (1.6), so that we may henceforth consider the sum (2.2). We write it as

$$
S=\sum_{M \leqslant m, n \leqslant M^{\prime}}(m n)^{-1 / 2}(m / n)^{i t} \sum_{j=1}^{\infty} \alpha_{j} h\left(\kappa_{j}\right) t_{j}(m) t_{j}(n),
$$


and apply in the inner sum the following Bruggeman-Kuznetsov sum formula (for a proof, see [Mo, Theorem 2.2]) which transforms a spectral sum into an arithmetic expression involving Kloosterman sums

$$
S(m, n ; \ell)=\sum_{a(\bmod \ell),(a, \ell)=1} e((a m+\bar{a} n) / \ell),
$$

where $a \bar{a} \equiv 1(\bmod \ell)$ and $e(\alpha)=e^{2 \pi i \alpha}$.

LEMMA 1. Let $h(r)$ be even and regular in the strip $|\operatorname{Im} r| \leqslant 1 / 2$, and there $|h(r)| \ll(1+|r|)^{-2-\varepsilon}$. Then we have, for any positive integers $m, n$,

$$
\begin{aligned}
\sum_{j=1}^{\infty} \alpha_{j} h\left(\kappa_{j}\right) & t_{j}(m) t_{j}(n)=-\frac{1}{\pi} \int_{-\infty}^{\infty} \frac{\sigma_{2 i r}(m) \sigma_{2 i r}(n)}{(m n)^{i r}|\zeta(1+2 i r)|^{2}} h(r) d r \\
+ & \frac{\delta_{m, n}}{\pi^{2}} \int_{-\infty}^{\infty} r h(r) \tanh (\pi r) d r+\sum_{\ell=1}^{\infty} \frac{1}{\ell} S(m, n ; \ell) h_{+}(4 \pi \sqrt{m n} / \ell)
\end{aligned}
$$

with

$$
h_{+}(x)=\frac{2 i}{\pi} \int_{0}^{\infty} \frac{r h(r)}{\cosh (\pi r)}\left(J_{2 i r}(x)-J_{-2 i r}(x)\right) d r .
$$

Here $\delta_{m, n}=1$ if $m=n$, and $\delta_{m, n}=0$ otherwise.

The contribution of the first term on the right of (2.4) to the sum $S$ is negative (because $\sigma_{2 i r}(n) n^{-i r}$ is real for real $r$ ) and can thus be neglected. Also, the $\delta$-term is easy, for its contribution is $\ll K G$.

In our treatment of the Kloosterman part in (2.4), we first truncate the sum over $\ell$ as in [JM1] (or [JM2]), namely to the range

$$
1 \leqslant \ell \ll \ell_{0}=M(G K)^{-1} \log K
$$

Indeed, the contribution of the remaining values of $\ell$ to $S$ is $\ll K^{-A}$, for any fixed positive $A$, which is negligible.

Next the integral transform $h_{+}(x)$ and the Kloosterman sum $S(m, n ; \ell)$ are substituted into (2.4), and the resulting expression is further substituted into (2.3), where the sums over $m$ and $n$ will be estimated for fixed $r, \ell$, and $a$.

At this stage, we need the following asymptotic expansion for the $J$-Bessel function (see [EMOT, Sec. 7.13.2, Eq. (17)]):

$$
\begin{gathered}
J_{2 i r}(x) \sim \frac{1}{\sqrt{2 \pi}}\left(x^{2}+4 r^{2}\right)^{-1 / 4} \exp (i \omega(r, x)+\pi r-\pi i / 4), \\
\omega(r, x)=\sqrt{4 r^{2}+x^{2}}-2 r \log \left(2 r / x+\sqrt{1+4(r / x)^{2}}\right)
\end{gathered}
$$

as $r$ and $x$ tend to infinity. The relation (2.6) gives the leading term of the expansion, and the subsequent terms not written down involve positive powers of 
$\left(x^{2}+4 r^{2}\right)^{-1 / 2}$ together with the same oscillatory factor; then the error term will be of the order $O\left(x^{-A}\right)$, where the positive number $A$ can be chosen arbitrarily large taken that the expansion is sufficiently long. This will be applied with $x$ larger than $r$, and then the first approximation to $\omega(r, x)$ is simply $x$, the second approximation is $x-2 r^{2} / x$, and so on. Suppose now that uniformly for all $a$ and $r \asymp K$ we have

$$
\sum_{m, n}(m / n)^{i t} e((a m+\bar{a} n) / \ell) \exp (\nu i \omega(r, 4 \pi \sqrt{m n} / \ell)) \ll T(\ell),
$$

where $\nu= \pm 1$ and $m, n$ run over any subintervals of $\left[M, M^{\prime}\right]$. Then, using (2.6) and two-dimensional summation by parts (see $[K$, Theorem 1.6]), we see that the contribution of the $\ell$-term in (2.4) to the sum $S$ is

$$
\ll M^{-3 / 2} G K \ell^{1 / 2} T(\ell) .
$$

For convenience, we formulate the two-dimensional partial summation formula.

Lemma 2. Let $f(m, n)$ and $g(m, n)$ be number theoretic functions and suppose that the partial derivatives $f_{x}, f_{y}$, and $f_{x y}$ are continuous in the rectangle $[1, X] \times$ $[1, Y]$. Put

$$
G(x, y)=\sum_{1 \leqslant m \leqslant x} \sum_{1 \leqslant n \leqslant y} g(m, n) .
$$

Then

$$
\begin{aligned}
& \sum_{1 \leqslant m \leqslant X} \sum_{1 \leqslant n \leqslant Y} f(m, n) g(m, n) \\
&=\int_{1}^{X} \int_{1}^{Y} G(x, y) f_{x y}(x, y) d y d x-\int_{1}^{X} G(x, Y) f_{x}(x, Y) d x \\
& \quad-\int_{1}^{Y} G(X, y) f_{y}(X, y) d y+G(X, Y) f(X, Y) .
\end{aligned}
$$

The double exponential sum in (2.7) can be estimated by the following lemma [K, Theorem 2.16] if $\ell$ is sufficiently large. The notation $A \asymp B$ means that the positive quantities $A$ and $B$ are of the same order of magnitude, that is $A \ll B$ and $B \ll A$.

Lemma 3. Suppose that

$$
\left|f_{x x}\right| \asymp \lambda_{1}, \quad\left|f_{y y}\right| \asymp \lambda_{2}, \quad\left|f_{x y}\right| \ll \sqrt{\lambda_{1} \lambda_{2}}, \quad\left|f_{x x} f_{y y}-f_{x y}^{2}\right| \gg \lambda_{1} \lambda_{2}
$$

throughout a rectangle $D=[A, A+M] \times[B, B+N]$ with $M, N \geqslant 1$. Then

$$
\begin{array}{r}
\sum_{(m, n) \in D} e(f(m, n)) \ll\left(M \lambda_{1}+N \sqrt{\lambda_{1} \lambda_{2}}+1\right)\left(N \lambda_{2}+M \sqrt{\lambda_{1} \lambda_{2}}+1\right)\left(\lambda_{1} \lambda_{2}\right)^{-1 / 2} \\
\times\left(1+\log (M N)+\left|\log \lambda_{1}\right|+\left|\log \lambda_{2}\right|\right) .
\end{array}
$$


In the case of the sum (2.7), we have

$$
f(x, y)=(t / 2 \pi) \log (x / y)+(a x+\bar{a} y) / \ell+(\nu / 2 \pi) \omega(r, 4 \pi \sqrt{x y} / \ell) .
$$

If now $\ell$ is sufficiently large, say $\ell \gg t^{\delta}$, then $\lambda_{1}, \lambda_{2} \asymp t / M^{2}$ since we have $M \ll t$. Also, $f_{x y} \ll(M \ell)^{-1}$ is of a lower order of magnitude than $\lambda_{1}$ and $\lambda_{2}$, so that the assumptions of Lemma 3 are satisfied, and it follows that $T(\ell) \ll t^{1+\varepsilon}$. Therefore the quantity (2.8) summed over $t^{\delta} \ll \ell \ll \ell_{0}$ is $\ll G K t^{1+\varepsilon} M^{-3 / 2} \ell_{0}^{3 / 2}$. By (2.1) and (2.5), this is $\ll t^{2 / 3+\varepsilon}$.

On the other hand, if $\ell \ll t^{\delta}$, then we estimate the double sum in (2.7) by Cauchy's inequality as $\ll M^{1 / 2}\left(\sum_{m}\left|\sum_{n}\right|^{2}\right)^{1 / 2}$. Here the square of the $n$-sum is written out as a double sum and the $m$-sum is taken inside. Then we end up with exponential sums of the type

$$
\sum_{m} e\left(\omega\left(r, 4 \pi \sqrt{m n_{1}} / \ell\right)-\omega\left(r, 4 \pi \sqrt{m n_{2}} / \ell\right)\right)
$$

The diagonal terms give a contribution $\ll M^{3 / 2}$ to the original double sum. Essentially the same comes from the non-diagonal terms, by a combination of van der Corput's lemma and the "first derivative test" ([Iv1, Lemmas 2.4 and 2.1]; the former is used in its simplest case). After a subdivision of the $n$-sum in (2.7), we may suppose that $\left|n_{1}-n_{2}\right| \leqslant c M$ for a suitably small positive constant $c$. In this way, we see that $T(\ell) \ll M^{3 / 2} t^{\delta}$. Then, by (2.8), the contribution of the the values $\ell \ll t^{\delta}$ to $S$ is $\ll G K t^{5 \delta / 2}$.

Remark. The above argument works in the case (1.5) as well, but then we get an additional term which is about of the order $t / K$ and exceeds thus $t^{2 / 3}$ for $t \gg K^{3}$. Therefore we need an alternative method in this case.

\section{The case $(1.5)$}

For $t \gg K^{3}$, the term $t^{2 / 3}$ dominates on the right of (1.6) whatever the size of $G$, so that it suffices to prove the estimate

$$
\sum_{K \leqslant \kappa_{j} \leqslant 2 K} \alpha_{j}\left|S_{j}\right|^{2} \ll t^{2 / 3+\varepsilon}
$$

with $S_{j}$ as in (1.7). By (1.8), we may suppose that

$$
t^{2 / 3+2 \delta} \ll M \ll t
$$

for some small $\delta>0$.

To begin with, we express the sum $S_{j}$ approximately in terms of certain short sums related to the rationals $\rho=a / q$ (in their lowest terms) with $Q \leqslant q \leqslant 2 Q$, where $Q>1$ is a parameter to be specified in a moment. In general, there are

$$
L=\sum_{Q \leqslant q \leqslant 2 Q} \varphi(q) \sim \frac{9 Q^{2}}{\pi^{2}}
$$


such numbers $\rho$ in the interval $[0,1]$. Therefore, statistically, one may expect that there are about $\lambda=L \eta$ numbers $\rho$ in an interval of length $\eta$ if $\eta \gg Q^{-2}$. We specify now $Q=M t^{-2 / 3-\delta}, \quad \eta=Q^{-1}$ noting that then $\lambda \asymp Q$. Let $u \geqslant 0$ be a "moothing parameter", the role of which will be to produce smooth weight functions by an averaging procedure, and define

$$
S_{j}(\rho, u)=\sum_{m \in I(\rho, u)} t_{j}(m) m^{-1 / 2-i t}
$$

where

$$
I(\rho, u)=\left[\frac{t}{2 \pi(\rho+2 \eta)}+u, \frac{t}{2 \pi(\rho+\eta)}+u\right] .
$$

In applications of the transformation method of [J1] to Dirichlet polynomials, the sum related to $\rho$ is usually chosen to be symmetric with respect to $t /(2 \pi \rho)$, but we prefer to work with the asymmetric ranges $I(\rho, u)$ in order to get a transformed sum of a more convenient form in the sense that all the indices of summation will be of the same order of magnitude.

The smoothing procedure in (3.3) runs as follows: writing $u=u_{1}+\cdots+u_{J}$ with $0 \leqslant u_{j} \leqslant U$, where $J$ a sufficiently large fixed positive integer and

$$
U=M t^{-1 / 2+\delta}
$$

we average $S_{j}(\rho, u)$ over the variables $u_{j}$ to construct a weighted sum, say $S_{j}(\rho)$. This device, used originally in [J1], improves the accuracy of the Voronoi transformation of exponential sums. But as yet we keep $u$ fixed, restrict $\rho$ to the interval

$$
R=\left[\frac{t}{2 \pi M^{\prime}}-\eta, \frac{t}{2 \pi M}-2 \eta\right]
$$

noting that then $I(\rho, u) \subseteq\left[M+u, M^{\prime}+u\right]$, and show next that the sum

$$
\tilde{S}_{j}(u)=\lambda^{-1} \sum_{\rho \in R} S_{j}(\rho, u)
$$

a linear combination of partially overlapping sums, is a good approximation to $S_{j}$ in the sense that

$$
\sum_{K \leqslant \kappa_{j} \leqslant 2 K} \alpha_{j}\left|S_{j}-\tilde{S}_{j}(u)\right|^{2} \ll t^{2 / 3+\varepsilon}
$$

uniformly for $0 \leqslant u \leqslant J U$.

To compare the sums $S_{j}$ and $\tilde{S}_{j}(u)$, we write their difference as

$$
S_{j}-\tilde{S}_{j}(u)=\lambda^{-1} \sum_{M \leqslant m \leqslant M^{\prime}+u} a_{m}(u) t_{j}(m) m^{-1 / 2-i t},
$$

and apply the spectral large sieve inequality, which we state as the following lemma (see [Mo, Theorem 3.3]). 
LEMma 4. For $C(\log K)^{1 / 2} \leqslant G \leqslant C^{-1} K(\log K)^{-1 / 2}$, where $C>0$ is sufficiently large constant, and for any complex vector $\{c(n)\}$ with $N \leqslant n \leqslant 2 N$, we have

$$
\sum_{K \leqslant \kappa_{j} \leqslant K+G} \alpha_{j}\left|\sum_{N \leqslant n \leqslant 2 N} c(n) t_{j}(n)\right|^{2} \ll\left(K G+N(\log K)^{3 / 2}\right) \sum_{N \leqslant n \leqslant 2 N}|c(n)|^{2} .
$$

Since $M \gg t^{2 / 3} \gg K^{2}$, we see that the left hand side of (3.6) is

$$
\ll \lambda^{-2} t^{\varepsilon} \sum_{M \leqslant m \leqslant M^{\prime}+u}\left|a_{m}(u)\right|^{2} .
$$

Now $m$ lies in the interval $I(\rho, u)$ with $\rho \in R$ whenever $\rho$ lies in the interval

$$
R \cap\left[\frac{t}{2 \pi(m-u)}-2 \eta, \frac{t}{2 \pi(m-u)}-\eta\right] .
$$

Therefore, for $m \in\left[M, M^{\prime}\right]$, the deviation from $\lambda$ of the number of our rationals $\rho$ in the above interval is measured by $\left|a_{m}(u)\right|$. The interval $R$ contains the latter interval in the intersection if

$$
M+u+2 \pi(m-u) M \eta t^{-1} \leqslant m \leqslant M^{\prime}+u-2 \pi(m-u) M^{\prime} \eta t^{-1} .
$$

This holds for all $m$ in (3.8) up to $\ll M t^{-1 / 3+\delta}$ exceptions. Since $a_{m}(u) \ll \lambda$ by the above remarks, the contribution of these exceptional values to $(3.8)$ is $\ll$ $M t^{-1 / 3+\delta+\varepsilon} \ll t^{2 / 3+2 \delta}$.

To the other values of $m$, we apply the following well-distribution property of rationals (see $[\mathrm{J} 3, \mathrm{~J} 4]$ ).

Lemma 5. Let $Q \geqslant 1, L=\sum_{Q \leqslant q \leqslant 2 Q} \varphi(q), 0<\Delta \leqslant 1 / 3, \lambda=2 \Delta L$, and let $\lambda(x)$ denote the number of the rationals $a / q$ with $(a, q)=1$ and $Q \leqslant q \leqslant 2 Q$ in the interval $[x-\Delta, x+\Delta]$. Then

$$
\int_{0}^{1}(\lambda(x)-\lambda)^{2} d x \ll \Delta Q^{2} \log ^{3}(1 / \Delta) .
$$

If we now interpret $a_{m}(u)$ statistically as above, we may view $m$ as a continuous variable and the sum over $m$ in (3.8) can be replaced by the corresponding integral with a small error. After a simple change of variable, this integral can be estimated by Lemma 5 with $\Delta=\eta / 2$ and $x$ running over an interval of length $\asymp t / M$; by periodicity, Lemma 5 readily implies an estimate even for integrals over intervals of length exceeding one. In this way, the remaining part of (3.8) is seen to be $\ll M Q^{-1} t^{\varepsilon} \log ^{3} Q \ll t^{2 / 3+2 \delta}$. Thus the inequality (3.6) is verified.

At this point, we average $S_{j}(\rho, u)$ over $u$ as explained above, constructing thus a smoothed sum which we denoted by $S_{j}(\rho)$. In other words, we average the sum $\tilde{S}_{j}(u)$ producing the sum

$$
\tilde{S}_{j}=\lambda^{-1} \sum_{\rho \in R} S_{j}(\rho)
$$


This is a good approximation to $S_{j}$ in the sense that (3.6) holds with $\tilde{S}_{j}$ in place of $\tilde{S}_{j}(u)$, and hence the inequality

$$
\sum_{K \leqslant \kappa_{j} \leqslant 2 K} \alpha_{j}\left|\tilde{S}_{j}\right|^{2} \ll t^{2 / 3+\varepsilon}
$$

implies the desired estimate (3.1). Thus it remains to prove (3.9).

Next we are going to transform the sums $S_{j}(\rho)$ as in [J1], that is by a summation formula of the Voronoi type, which in the present case reads as follows (see [Me1, Theorem 2]): if $f \in C^{1}[A, B]$, then

$$
\begin{aligned}
& \text { (3.10) } \sum_{A \leqslant n \leqslant B}^{\prime} t_{j}(n) e\left(\frac{a n}{q}\right) f(n) \\
& =\frac{\pi i}{q \sinh \pi \kappa_{j}} \sum_{n=1}^{\infty} t_{j}(n) e\left(-\frac{\bar{a} n}{q}\right) \int_{A}^{B}\left\{J_{2 i \kappa_{j}}\left(\frac{4 \pi}{q} \sqrt{n x}\right)-J_{-2 i \kappa_{j}}\left(\frac{4 \pi}{q} \sqrt{n x}\right)\right\} f(x) d x \\
& \quad+4 \epsilon_{j} q^{-1} \cosh \pi \kappa_{j} \sum_{n=1}^{\infty} t_{j}(n) e\left(\frac{\bar{a} n}{q}\right) \int_{A}^{B} K_{2 i \kappa_{j}}\left(\frac{4 \pi}{q} \sqrt{n x}\right) f(x) d x
\end{aligned}
$$

where $\epsilon_{j}$ is the parity sign of the $j$ th Maass form. The convention of summation on the left is that if $A$ or $B$ is an integer, then the corresponding term is to be halved. We apply this with $-\rho=-a / q$ in place of $a / q$ and with $f(x)=x^{-1 / 2-i t} e(\rho x)$; the role of the factor $e(\rho x)$ here is to damp the oscillations of $x^{-i t}$. The range of summation is the interval $I(\rho, u)$, or ultimately the interval

$$
I(\rho)=\left[\frac{t}{2 \pi(\rho+2 \eta)}, \frac{t}{2 \pi(\rho+\eta)}+J U\right]
$$

once the smoothing has been done.

The above argument yields a transformation formula for the sum $S_{j}(\rho)$ if the Bessel functions are expressed by their asymptotic formulae and the integrals are approximately evaluated by the saddle point method. In fact, such a formula has been worked out by T. Meurman (see [Me2]). On the other hand, analogous but somewhat simpler transformation formulae for Dirichlet polynomials involving the divisor function $d(n)$ or Fourier coefficients of holomorphic cusp forms can be found in [J1], and to avoid complications we want to utilize the latter results.

In our case, the dominating contribution will come from the $J$-Bessel part of (3.9), and moreover from a suitable sum over $n \in\left[c_{1} M, c_{2} M\right]$ for some positive constants $c_{i}$, with $c_{1}$ sufficiently small and $c_{2}$ sufficiently large, for otherwise the respective integrals will be negligible by the general principle that an exponential integrals with a smooth weight function and without a saddle point is small.

The strategy of such a "comparison argument" is as follows. The dominating contribution in (3.10) will come from the $J$-Bessel part, and the integrals will be calculated by the saddle point method. In our case, the oscillatory part of the asymptotic formula (2.6) for the $J$-Bessel functions with $r=\kappa_{j}$ can be made 
independent of $\kappa_{j}$ since it is sufficiently small compared with $x$, and then the $J$-integrals in (3.10) will be approximately of the same structure as those in the corresponding formula involving $d(n)$ in place of $t_{j}(n)$, as far as the leading term of the saddle point method is concerned. The analogue of (3.10) for a sum involving $d(n)$ in place of $t_{j}(n)$ (see [J1, Theorem 1.7]) is a generalization of Voronoi's classical summation formula, and it will serve as a simplified model for us. This formula involves the Bessel function $Y_{0}(x)$, and in fact the same function emerges in (3.10) if we let heuristically $\kappa_{j}$ tend to zero. Indeed, by the definition of $Y_{0}(x)$, we have

$$
Y_{0}(x)=\lim _{r \rightarrow 0} \frac{J_{2 i r}(x)-J_{-2 i r}(x)}{i \sinh (2 \pi r)},
$$

so that

$$
\begin{array}{r}
\frac{\pi i}{q \sinh \pi \kappa_{j}} \int_{A}^{B}\left\{J_{2 i \kappa_{j}}\left(\frac{4 \pi}{q} \sqrt{n x}\right)-J_{-2 i \kappa_{j}}\left(\frac{4 \pi}{q} \sqrt{n x}\right)\right\} f(x) d x \\
\approx-\frac{2 \pi}{q} \int_{A}^{B} Y_{0}\left(\frac{4 \pi}{q} \sqrt{n x}\right) f(x) d x
\end{array}
$$

For the clarity of presentation, we postpone the justification of this approximation and the omission of the other terms in (3.10) to the next section. As we mentioned above, the point is that the leading explicit terms on both sides of (3.12) will coincide in our case, and these terms will play a dominating role in the proof of (3.9).

To transform the smoothed subsum $S_{j}(\rho)$ according to the comparison principle, we introduce some notation. Write the interval (3.11), that is the range of $S_{j}(\rho)$, as

$$
I(\rho)=\left[M_{1}, M_{2}\right]=\left[\frac{t}{2 \pi \rho}-\mu_{1}, \frac{t}{2 \pi \rho}-\mu_{2}\right],
$$

put $N=a^{2} \mu_{2}^{2} M_{2}^{-1}, N^{\prime}=a^{2} \mu_{1}^{2} M_{1}^{-1}$, let $\chi(s)$ be in the functional equation $\zeta(s)=$ $\chi(s) \zeta(1-s)$, and define

$$
\phi(x)=\operatorname{arsinh}\left(x^{1 / 2}\right)+\left(x+x^{2}\right)^{1 / 2} .
$$

Note that $\mu_{i} \asymp t \eta \rho^{-2} \asymp M^{2} Q^{-1} t^{-1}=M t^{-1 / 3+\delta}$ and $a \asymp M^{-1} Q t$, so that $N, N^{\prime} \asymp$ $M$.

Then, assuming for a moment that the approximation (3.12) holds and that the $K$-Bessel part in the summation formula is negligible, we may quote from [J1], Theorem 4.3, the following leading explicit part of the transformation formula for $S_{j}(\rho)$ (it is easy to check that the conditions of that theorem are satisfied, in particular the condition $\mu_{i} \gg a q t^{\varepsilon}$ holds by our choice of $Q$ ):

$$
\begin{aligned}
S_{j}(\rho) \sim \pi^{1 / 4}(2 a q t)^{-1 / 4} \rho^{i t} \chi\left(\frac{1}{2}+i t\right) \sum_{N \leqslant n \leqslant N^{\prime}} t_{j}(n) w(n, \rho) \\
\quad \times e\left(n\left(\frac{\bar{a}}{q}-\frac{1}{2 a q}\right)\right) n^{-1 / 4}\left(1+\frac{\pi n}{2 a q t}\right)^{-1 / 4} \exp \left(2 i t \phi\left(\frac{\pi n}{2 a q t}\right)+\frac{\pi}{4}\right),
\end{aligned}
$$


where $w(n, \rho)$ is a piecewise smooth bounded weight function. To read the above sum literally from [J1], one may view the sum $S_{j}(\rho)$ as the difference of two more symmetric sums (with respect to the point $t /(2 \pi \rho)$ ) and take then the difference of the transformed sums. Of course, there is nothing to correspond to the leading term in Theorem 4.3, for $t_{j}(n)$, unlike $d(n)$, is a function of oscillating sign. The error term in $[\mathrm{J} 1]$ is

$$
\ll a^{2} q^{-1} \mu_{i}^{1 / 2} t^{-3 / 2} U \log t \ll Q(t / M)^{2}\left(M t^{-1 / 3+\delta}\right)^{1 / 2} t^{-3 / 2} U \log t \ll M^{1 / 2} t^{-5 / 6+\delta}
$$

with $U$ as in (3.4), and its proof makes use of well-known properties of the divisor function. Though a full analogy between the estimates for $d(n)$ and $t_{j}(n)$, that is the Ramanujan-Petersson conjecture, is still an open problem, it suffices for our purposes that $t_{j}(n)$ is known to be essentially bounded in mean, and this property follows in fact from the estimate

$$
\sum_{n \leqslant x} t_{j}^{2}(n) \ll \kappa_{j}^{\varepsilon} x
$$

due to H. Iwaniec (see [Iw2, Lemma 1]). The contribution of the above error terms to the sum $\tilde{S}_{j}$ is $\ll Q(t / M) M^{1 / 2} t^{-5 / 6+\delta} \ll(M / t)^{1 / 2} \ll 1$, which is negligible.

We substitute now the preceding approximate formula for $S_{j}(\rho)$ to the definition of $\tilde{S}_{j}$ getting a double sum over $n$ and $\rho$. Then, using the spectral large sieve, we reduce the sum (3.9) to the estimation of sums of the type

$$
\begin{array}{rl}
t M^{-2} \sum_{a_{1} / q_{1}} \sum_{a_{2} / q_{2}} \mid \sum_{n \asymp M} & e\left(n\left(\frac{\overline{a_{1}}}{q_{1}}-\frac{\overline{a_{2}}}{q_{2}}-\frac{1}{2 a_{1} q_{1}}+\frac{1}{2 a_{2} q_{2}}\right)\right) \\
& \times \exp \left(2 i t\left(\phi\left(\frac{\pi n}{2 a_{1} q_{1} t}\right)-\phi\left(\frac{\pi n}{2 a_{2} q_{2} t}\right)\right)\right) \mid
\end{array}
$$

we applied partial summation to simplify the $n$-sum and used the estimate $\lambda^{-2}(\text { aqtn })^{-1 / 2} \ll M^{-3} t^{1+3 \delta}$.

Since the number of the relevant rationals $\rho$ is $\ll Q^{2}(t / M) \ll M t^{-1 / 3-2 \delta}$, the diagonal terms contribute $\ll\left(t M^{-2}\right)\left(M t^{-1 / 3-2 \delta}\right) M \ll t^{2 / 3}$.

Turning to the non-diagonal terms, we classify these into $\ll \log ^{2} t$ groups either according to the conditions

$$
\begin{aligned}
|| \frac{\overline{a_{1}}}{q_{1}}-\frac{\overline{a_{2}}}{q_{2}} \| & \asymp \Delta_{1}, \\
\left|a_{1} q_{1}-a_{2} q_{2}\right| & \asymp \Delta_{2} M t^{-1 / 3-2 \delta}
\end{aligned}
$$

for some positive numbers $\Delta_{i}$, or allowing the right hand side of (3.14) or (3.15) vanish so that we may put $\Delta_{1}=0$ or $\Delta_{2}=0$ (both of them cannot vanish simultaneously outside the diagonal). As usual, $\|\alpha\|$ denotes the distance of $\alpha$ from the nearest integer. The number of such pairs $\left(a_{1} / q_{1}, a_{2} / q_{2}\right)$ can be estimated by the following well-known lemma of E. Bombieri and H. Iwaniec (for a proof of a generalized version, see $[\mathrm{HW}])$ : 
LEMMA 6. The number of the pairs of rationals $\left(a_{1}, q_{1}, a_{2} / q_{2}\right)$ satisfying $a_{i} \asymp$ $A, q_{i} \asymp Q$, and

$$
\begin{gathered}
|| \frac{\overline{a_{1}}}{q_{1}}-\frac{\overline{a_{2}}}{q_{2}} \| \leqslant \Delta_{1}, \\
\left|a_{1} q_{1}-a_{2} q_{2}\right| \leqslant \Delta_{2} A Q
\end{gathered}
$$

with $\Delta_{1}, \Delta_{2} \geqslant 0$ is

$$
\ll A Q+\Delta_{2}\left(A^{2}+Q^{2}\right)+\Delta_{1}\left(\Delta_{1}+\Delta_{2}\right) A^{2} Q^{2} .
$$

The $n$-sum in (3.13) is estimated by van der Corput's lemma [Iv1, Lemma 2.4], that is by the corresponding integral and a bounded term in our case. To this end, we have to analyse the oscillatory nature of this sum. Note that

$$
\phi^{\prime}(x)=\left(\frac{1+x}{x}\right)^{1 / 2} .
$$

In (3.13), we write the summand as $e(f(n)$ with

$$
f(n)=n\left(\frac{\overline{a_{1}}}{q_{1}}-\frac{\overline{a_{2}}}{q_{2}}\right)+g(n)
$$

and

$$
g(n)=n\left(-\frac{1}{2 a_{1} q_{1}}+\frac{1}{2 a_{2} q_{2}}\right)+(t / \pi)\left(\phi\left(\frac{\pi n}{2 a_{1} q_{1} t}\right)-\phi\left(\frac{\pi n}{2 a_{2} q_{2} t}\right)\right) .
$$

Then for $x \asymp M, q_{i} \asymp M t^{-2 / 3-\delta}, a_{i} \asymp t^{1 / 3-\delta}$, and assuming (3.15) (possibly for $\Delta_{2}=0$, meaning that the left hand side vanishes), we have

$$
\begin{gathered}
f^{\prime}(x)=\frac{\overline{a_{1}}}{q_{1}}-\frac{\overline{a_{2}}}{q_{2}}+\theta \Delta_{2} t^{2 / 3+\delta} M^{-1}, \\
f^{\prime \prime}(x)=g^{\prime \prime}(x) \asymp \Delta_{2} t^{2 / 3+\delta} M^{-2},
\end{gathered}
$$

where $\theta \asymp 1$. Note that the second term in (3.17) is $\ll t^{-\delta}$ by $(3.2)$.

A saddle point $x_{0}$ satisfying $f^{\prime}\left(x_{0}\right)=0$ and $x_{0} \asymp M$ can possibly exist only if

$$
\Delta_{2} t^{2 / 3+\delta} M^{-1} \asymp \Delta_{1}
$$

In the case (3.19) we use the "econd derivative test" [Iv1, Lemma 2.2] and (3.18) to show that the $n$-sum is $\ll M t^{-1 / 3-\delta / 2} \Delta_{2}^{-1 / 2}$, and otherwise the above mentioned "first derivative test" is applicable.

Let us consider the case (3.19) first. Then $\Delta_{1}<\Delta_{2}$, and for the quantity (3.16), say $B$, we have the estimate

$$
B \ll M t^{-1 / 3}+\Delta_{2} t^{2 / 3}+\Delta_{1} \Delta_{2} M^{2} t^{-2 / 3-4 \delta} \ll M t^{-1 / 3}+\Delta_{2} t^{2 / 3}+\Delta_{2}^{2} M,
$$


where we ignored some unimportant $\delta$-factors for simplicity. We may suppose that $\Delta_{2} \gg t^{-1 / 3}$, for otherwise the right hand side of (3.20) will be as in the case of the diagonal terms. By the second derivative test, we get the contribution

$$
\ll\left(t M^{-2}\right)\left(M t^{-1 / 3} \Delta_{2}^{-1 / 2}\right) B \ll t^{1 / 3} \Delta_{2}^{-1 / 2}+t^{4 / 3} M^{-1} \Delta_{2}^{1 / 2}+t^{2 / 3} \Delta_{2}^{3 / 2} \ll t^{2 / 3}
$$

to the sum (3.13).

We are now left with the case $\left|f^{\prime}(x)\right| \asymp r$, where $r=\Delta_{1}$ or $r=\Delta_{2} t^{2 / 3+\delta} M^{-1}$ depending on which one of the terms on the right of (3.17) dominates, and we have to estimate the quantity

$$
t M^{-2} B r^{-1}
$$

First, if $r=\Delta_{2} t^{2 / 3+\delta} M^{-1}$, then $\Delta_{1}<\Delta_{2}$ as above, and $B$ can be estimated as in (3.20). Hence (3.21) is

$$
\ll \Delta_{2}^{-1}+t / M+\Delta_{2} t^{1 / 3} \ll t^{1 / 3},
$$

since again we may suppose that $\Delta_{2} \gg t^{-1 / 3}$.

Finally, if $r=\Delta_{1}$, then we have $\Delta_{1} \gg \Delta_{2} t^{2 / 3+\delta} M^{-1}$ and

$$
B \ll M t^{-1 / 3}+\Delta_{1} M+\Delta_{1}^{2} M^{3} t^{-4 / 3} .
$$

Hence the quantity (3.21) is

$$
\ll t^{2 / 3}\left(M \Delta_{1}\right)^{-1}+t M^{-1}+\Delta_{1} M t^{-1 / 3} .
$$

Here we may suppose that $M \Delta_{1} \gg t^{1 / 3}$ since obviously a natural lower bound for the positive values of $\Delta_{1}$ is $\Delta_{1} \gg Q^{-2}=t^{4 / 3+2 \delta} M^{-2}$. Hence we get again the estimate $\ll t^{2 / 3}$.

\section{The structure of the transformed sums}

In the preceding section, it remained to be verified that if the sum $S_{j}(\rho)$ is transformed by the summation formula (3.10), then the functional structure of the transformed sum will be independent of $\kappa_{j}$, as far as the "first approximation" is concerned, if $\kappa_{j}$ is sufficiently small compared with $t$, namely $\kappa_{j} \ll t^{1 / 3-\varepsilon}$.

We establish this structural invariance by showing that both sides of (3.12) give the same leading term, and the other terms in this approximation are of similar structure but of smaller order of magnitude. Because we are interested in estimations, only the leading term is significant.

The comparison of both sides of (3.12) amounts to the comparison of the respective Bessel functions, for which we have the asymptotic formulae

$$
Y_{0}(x) \sim\left(\frac{2}{\pi x}\right)^{1 / 2} \sin \left(x-\frac{\pi}{4}\right)
$$




$$
\frac{J_{2 i r}(x)}{\sinh \pi r} \sim\left(\frac{2}{\pi x}\right)^{1 / 2} e^{i x-i \pi / 4}
$$

by (2.6), the latter asymptotic formula holds if $r$ is large and $r \ll x^{1 / 2-\varepsilon}$, and this condition holds by (1.5) and and our choice $Q=M t^{-2 / 3-\delta}$ if $r \asymp K$ and $x \asymp M / Q$. We used here the approximation $\omega(r, x)=x-2 r^{2} / x+\cdots$ noting that the function $\exp \left(-2 i r^{2} / x+\cdots\right)$ can be written as a descending series with leading term 1 . Now, since the leading term on the right of (4.1) is independent of $r=\kappa_{j}$, the same is true for the first approximations of the respective transformed sums for various values of $\kappa_{j}$.

In the preceding analysis we had $n$ in a suitable interval $\left[c_{1} M, c_{2} M\right]$ with $c_{1}$ a sufficiently small and $c_{2}$ a sufficiently large positive constant. In the case $n>c_{2} M$, the same comparison principle works, and as in [J1], the corresponding $J$-Bessel terms will be small because then the integrals in the summation formula have no saddle point. The same is true also for $n<c_{1} M$; now we have to appeal again to the formula (2.6).

Finally, to deal with the $K$-Bessel terms in (3.10), we apply the following formula (see [L, p. 140]):

$$
K_{2 i r}(y)=\frac{2^{2 i r} \Gamma\left(\frac{1}{2}+2 i r\right)}{y^{2 i r} \sqrt{\pi}} \int_{0}^{\infty} \frac{\cos y s}{\left(1+s^{2}\right)^{2 i r+1 / 2}} d s .
$$

By use of Stirling's formula and a saddle-point argument, we see that the function $\cosh (\pi r) K_{2 i r}(y)$ decays rapidly if $y$ exceeds the "transitional point" $2 r$ since then there is no saddle point in the $s$-integral and one may integrate repeatedly by parts with respect to the trigonometric factor in the integrand. In our case, with $r=\kappa_{j}$ and $y=4 \pi \sqrt{n x} / q$, the transitional value occurs for

$$
n \asymp n_{0}=K^{2} Q^{2} / M=K^{2} M t^{-4 / 3-2 \delta} .
$$

By the above remark, the $n$-sum can be truncated to $n \leqslant n_{0} t^{\varepsilon}$.

By (4.2), the dependence of $K_{2 i \kappa_{j}}(4 \pi \sqrt{n x} / q)$ on $x$ is given by the function

$$
x^{-i r} \exp ( \pm 4 \pi i s \sqrt{n x} / q),
$$

and using again a saddle-point argument we may restrict $s$ to $s \ll K Q / \sqrt{n M}+1$. If the function (4.3) is written as $e(i \varphi(x))$, then the occurrence of a saddle point for the $K$-Bessel integrals in (3.10) with $f(x)=x^{-1 / 2-i t} e(\rho x)$ (times a smooth weight function) and $[A, B]=I(\rho)$ would require that $\left|\varphi^{\prime}(x)\right|$ should be comparable with $|\rho-t /(2 \pi x)|$, that is with $\eta=1 / Q=t^{2 / 3+\delta} / M$. But we have

$$
\varphi^{\prime}(x) \ll K / M+(K Q / \sqrt{n M}+1) \sqrt{n / M} Q^{-1} \ll(K / M) t^{\varepsilon} \ll t^{1 / 3+\varepsilon} / M,
$$

so that no saddle points occur, and therefore the contribution of the $K$-Bessel functions to (3.10) is negligible. 


\section{References}

[EMOT] A. Erdélyi, W. Magnus, F. Oberhettinger, and F. Tricomi, Higher Trancendental Functions, vol. 2, McGraw-Hill, New York-Toronto-London, 1953.

$[\mathrm{HW}]$ M. N. Huxley and N. Watt, Exponential sums and the Riemann zeta function, Proc. London Math. Soc. (3) 57 (1988), 1-24.

[Iv1] A. Ivić, The Riemann Zeta-Function, John Wiley \& Sons, New York, 1985; reprinted by Dover Publications, 2003.

[Iv2] A. Ivić, On sums of Hecke series in short intervals, J. Théorie des Nombres de Bordeaux 13 (2001), 554-568.

[Iw1] H. Iwaniec, Small eigenvalues of Laplacian for $\Gamma_{0}(N)$, Acta Arith. 56 (1990), 65-82.

[Iw2] H. Iwaniec, The spectral growth of automorphic L-functions, J. Reine Angew. Math. 428 (1992), 139-159.

[J1] M. Jutila, Lectures on a method in the theory of exponential sums, Tata Institute Lectures in Mathematics and Physics 80, Springer-Verlag, Bombay, 1987.

[J2] M. Jutila, The fourth power moment of the Riemann zeta-function over a short interval, Coll. Math. Soc. János Bolyai 51. Number Theory, Budapest 1987, North-Holland, 1989, pp. 221-244.

[J3] M. Jutila, Transformations of exponential sums, Proc. Amalfi Conf. Analytic Number Theory (Maiori 1989), University of Salerno, Salerno, 1992, pp. 263-270.

[J4] M. Jutila, The fourth moment of central values of Hecke series, Number Theory, Proc. of the Turku Symposium on Number Theory in Memory of Kustaa Inkeri, eds. Matti Jutila and Tauno Metsänkylä, Walter de Gruyter, Berlin-New York, 2001, pp. 167-177.

[J5] M. Jutila, Distribution of rational numbers in short intervals, The Ramanujan J. (to appear).

[JM1] M. Jutila and Y. Motohashi, A note on the mean value of the zeta and L-functions. XI, Proc. Japan Academy Ser. A, 78:1 (2002), 1-6.

[JM2] M. Jutila and Y. Motohashi, Uniform bound for Hecke L-functions (to appear).

[K] E. Krätzel, Lattice Points, Kluver Academic Publishers, Dordrecht-Boston-London, 1988.

[L] N. N. Lebedev, Special Functions and their Applications, Dover, 1972.

[Me1] T. Meurman, On exponential sums involving the Fourier coefficients of Maass wave forms, J. Reine Angew. Math. 384 (1988), 192-207.

[Me2] T. Meurman, On the order of the Maass L-function on the critical line, Coll. Math. Soc. János Bolyai 51. Number Theory, Budapest 1987, North-Holland, 1989, pp. 325-354.

[Mo] Y. Motohashi, Spectral Theory of the Riemann Zeta-Function, Cambridge University Press, 1997.

Department of Mathematics

(Received 0505 2005)

University of Turku

(Revised 01112004 )

FIN-20014 Turku

Finland

jutila@utu.fi 